\title{
Detection of Outer Wall Defects on Steel Pipe Using an Encircling Rotating Electromagnetic Field Eddy Current (RoFEC) Technique
}

\author{
Xiaokang Yin* - Zhuoyong Gu - Wei Wang - Xiaorui Zhang - Xin'an Yuan -Wei Li - Guoming Chen \\ Centre for Offshore Engineering and Safety Technology, China University of Petroleum (East China), China
}

In recent years, the rotating electromagnetic field eddy current (RoFEC) testing technique has attracted widespread attention due to its various advantages for inspecting tubular structures. However, most of the related work was focused on detecting inner wall defects on metal pipes using feed-through probes, which are often not applicable for outer wall defect detection. This work pushes forward the encircling RoFEC technique and demonstrates its feasibility for detecting outer wall defects. First, the basic principle of the encircling RoFEC technique is introduced. A three-dimensional finite element (FE) model was built in COMSOL to analyse the distribution of the rotating electromagnetic field and study the interaction between the defects and eddy currents. The axial component of the resultant magnetic field due to defects was selected as a characteristic signal and obtained from the FE models to study the factors, including pipe tilt, defect circumferential location, defect orientation and defect size, that influence the detection performance. An encircling RoFEC system using a probe with six excitation windings and a single bobbin pickup coil was constructed and used to inspect a steel pipe with one axial and one circumferential defect. The obtained voltage signal due to defects can form a Lissajous pattern in the impedance plane and be used for defect evaluation. The results showed that the encircling RoFEC technique can detect outer wall defects of both orientations and determine the circumferential location of the defect.

Keywords: non-destructive testing, rotating electromagnetic field, finite element, influencing factors

Highlights

- An encircling rotating electromagnetic field eddy current (RoFEC) technique is proposed to detect outer wall defects on a steel pipe.

- The encircling RoFEC technique is capable of detecting defects of arbitrary orientations on a steel pipe and determining its circumferential location.

- Finite element models are used to study the effects of pipe tilt, defect location, defect orientation and defect size on the inspection performance.

- An encircling RoFEC system was developed and used to inspect a steel pipe with both axial and circumferential defects to verify the feasibility of the proposed technique.

\section{O INTRODUCTION}

Steel pipes are widely used in the petroleum industry. In the process of long-term service, steel pipes are prone to defects, such as deformation, corrosion, and cracks [1], leading to the possible leakage of the conveying medium and/or failure of the whole structure [2]. Extensive efforts have been presented into the nondestructive evaluation (NDE) techniques capable of detecting defects in steel pipes, such as eddy current inspection [3] to [5], magnetic flux leakage inspection [6] and [7], ultrasonic guided wave inspection [8] and [9], etc. The above-mentioned techniques have been widely used in steel pipe inspection, but some of them have inherent limitations, e.g., traditional eddy current can only detect defects in the area under the probe; thus, circumferential and axial scanning are required to achieve full coverage; Some detection techniques, such as magnetic flux leakage and alternating current field measurement (ACFM), are more sensitive to cracks in certain directions. There is an increasing need for electromagnetic NDE methods that can thoroughly detect cracks in arbitrary directions, and many novel techniques have been proposed recently, such as circumferential eccentric eddy current probe [10], the array eddy current testing probe [11], etc. In recent years, the rotating electromagnetic field eddy current (RoFEC) method [12] has emerged as a useful NDE technique for plate-like structures [13] to [15], tubular structures [16] to [18], and welding seams [19] and [20].

Due to its unique advantages, e.g., the RoFEC technique is sensitive to defects of all orientations, it relies on the rotation of the probing field rather than the mechanical scan of the sensor to implement circumferential scan, and the phase information of the resultant signal can be used to determine the circumferential location of the defect, the RoFEC technique is particularly suitable for tubular structure inspection. A literature review reveals that the majority of related research focused on the detection of inner wall defects on pipes using feed-through probes 
[19] to [22] and using the RoFEC technique for the detection of outer wall defects on steel pipes can only be found sporadically in literature. It is thus necessary to embark in-depth research on the feasibility of encircling RoFEC technique on the detection of outer wall defect on steel pipes, as for some steel tubular structures, e.g., the widely used coiled tubing in the oil and gas industry, defects on the outer wall are commonly seen and need to be detected.

For the detection of outer wall defects on steel pipes, this paper pushes forward the encircling RoFEC technique by systematically studying the influential factors. The working principle of the encircling RoFEC technique is presented. Three-dimensional finite element (FE) models were constructed to demonstrate the perturbation of the probing field due to defects. The effects of different influencing factors on the detection results of the external penetrating rotating electromagnetic field detection sensor are explored, the encircling RoFEC system is built, and the detection test of a pipe with two typical flaws is carried out. The experimental results are consistent with the finite element simulation results and used to verify the feasibility of the proposed technique.

\section{PRINCIPLE OF THE ENCIRCLING ROFEC TECHNIQUE}

Unlike conventional feed-through RoFEC probes [23] to [25], the proposed encircling RoFEC probe comprises at least three external excitation windings and a single receiving coil. The three excitation windings are evenly distributed (120 degrees apart) around the pipe in space, as shown in Fig. 1a. The windings are driven by a three-phase sinusoidal current source. Currents in the three windings are identical in amplitude, but $120^{\circ}$ apart in phase angle, as shown in Eq. (1),

$$
\left\{\begin{array}{l}
i_{A}=I \sin \omega t \\
i_{B}=I \sin \left(\omega t-\frac{2}{3} \pi\right) \\
i_{C}=I \sin \left(\omega t+\frac{2}{3} \pi\right), \\
i_{X}=-i_{A} \\
i_{Y}=-i_{B} \\
i_{Z}=-i_{C}
\end{array}\right.
$$

where $i_{A}, i_{B}$ and $i_{C}$ correspond to the incoming sinusoidal alternating current of winding $\mathrm{A}$, winding $\mathrm{B}$, and winding $\mathrm{C}$ in Fig. 1a, respectively. Their amplitudes are the same, but the phase angles are $120^{\circ}$ apart. $i_{X}, i_{Y}$ and $i_{Z}$ are the outgoing currents of winding $\mathrm{A}$, winding $\mathrm{B}$ and winding $\mathrm{C}$, respectively. $I$ is the amplitude of sinusoidal alternating current, in [A], and $\omega$ is the frequency of the sinusoidal alternating current, in $[\mathrm{Hz}]$.

The magnetic fields $(B a, B b$, and $B c)$ generated by the windings are perpendicular to the plane of each winding, as shown in Fig. 1a. According to Eq. (1), the magnetic fields vary sinusoidally with $120^{\circ}$ apart in phase angle. The three components synthesize a magnetic field $\mathbf{B}$ with a constant amplitude and rotate circumferentially at a speed related to the excitation voltage frequency, similar to that found in a threephase induction motor. The rotating magnetic field B can then induce eddy currents that flow circularly around the radial axis. As shown in Fig. 1b, defects in any direction can disturb the eddy currents, thereby perturbing the secondary magnetic field, which can then be measured and used for defect evaluation. Note that the eddy currents also rotate circumferentially, offering full coverage of the pipe wall circumference.

a)
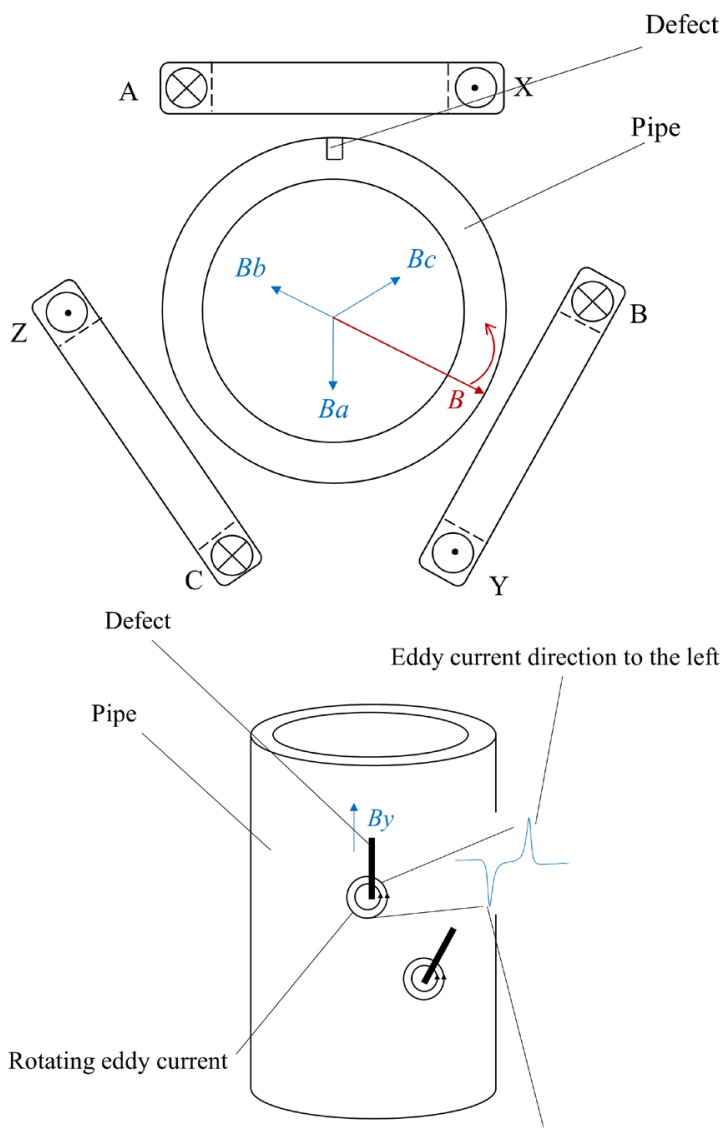

b)

Eddy current direction to the right

Fig. 1. The FE model of rotating magnetic field;

a) rotating magnetic field generation, and

b) interaction of eddy currents and defects 


\section{FE MODELLING OF THE ENCIRCLING ROFEC PROBE}

\subsection{The 3D FE Model}

A three-dimensional FE model of the encircling RoFEC probe was built in COMSOL and shown in Fig. 2. In the FE model, six excitation windings were used for more evenly distributed rotating fields. The windings are evenly distributed around the pipe, and the material of the pipe is steel. The geometric parameters of the FE model and the characteristic parameters of the coil are shown in Tables 1 and 2 . Fig. 3 shows the rotating magnetic field in the crosssection plane at different points in time $(T / 6, T / 3$, and $T / 2$ ). $T$ here refers to the time spent in a rotation cycle, that is, the time spent rotating the magnetic field around the tube, which is a fixed value.

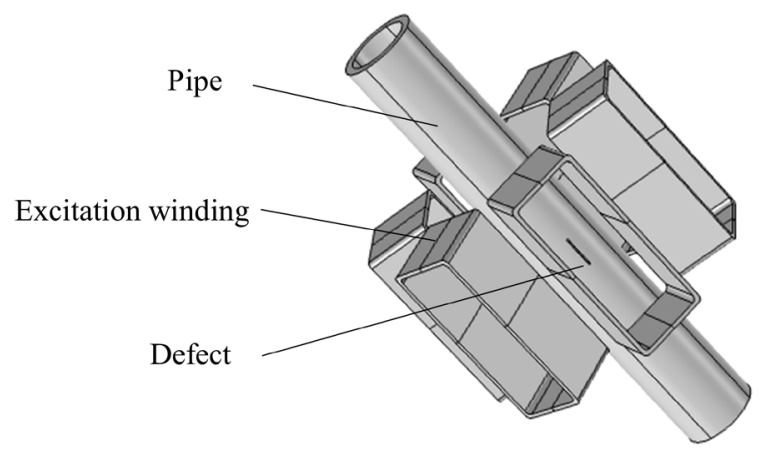

Fig. 2. The FE model of rotating magnetic field

Table 1. Geometric parameters of the FE model

\begin{tabular}{lc}
\hline Finite element model & Size [mm] \\
\hline Inside and outside diameter of pipe & $40 / 50$ \\
\hline Length of pipe & 150 \\
\hline Outer width of excitation winding & 45 \\
\hline Inner width of excitation winding & 40 \\
\hline Length of excitation winding & 115 \\
\hline Thickness of excitation winding & 40 \\
\hline Number of turns of the excitation windings & 100 \\
\hline Length of defect & 25 \\
\hline Width of defect & 1 \\
\hline Air domain diameter & 200 \\
\hline
\end{tabular}

Table 2. Characteristic parameters of the excitation windings

\begin{tabular}{cccc}
\hline $\begin{array}{c}\text { Coil wire } \\
\text { diameter [mm] }\end{array}$ & Coil turns & $\begin{array}{c}\text { Excitation } \\
\text { voltage [V] }\end{array}$ & $\begin{array}{c}\text { Excitation } \\
\text { frequency }[\mathrm{kHz}]\end{array}$ \\
\hline 1 & 100 & 0.5 & 10 \\
\hline
\end{tabular}

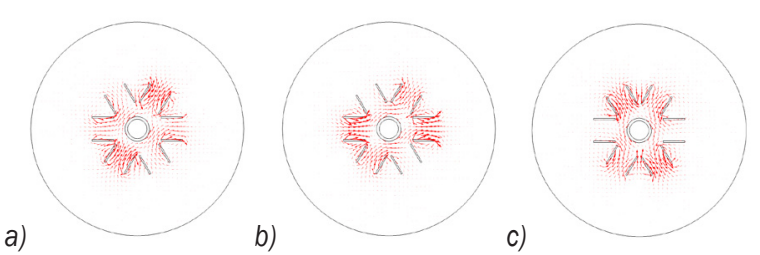

Fig. 3. Magnetic field in the $x=0$ plane at different moments; a) time $T / 6, b)$ time $T / 3$, and c) time $T / 2$

\subsection{Characteristic Signal due to Defect}

The excitation coil generates eddy current on the surface of the pipe, which also rotates around the axis at a certain speed depending on the frequency of the excitation. As shown in Fig. 4, when the eddy current encounters a defect (e.g., an axial crack), it will bypass each end of the defect and generate a secondary magnetic field. The resultant secondary magnetic field can then be measured and used to form indications of the defect. In theory, all components of the secondary magnetic field (axial, radial, and circumferential) can be used for defect detection and characterization. In this paper, the axial component was selected, as it can be easily measured by a single bobbin pickup coil. The advantages of such a measurement scheme will be further analysed in the experimental section.

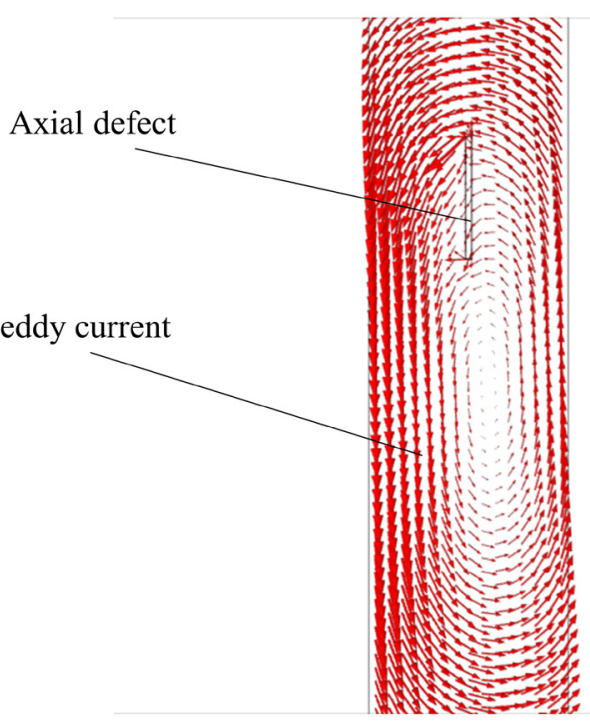

Fig. 4. Interaction of eddy currents and defects

In the FE model, the defect was moved across the windings to simulate an axial scan, and the axial magnetic field at $1 \mathrm{~mm}$ above the defect is extracted and plotted as the characteristic signals, as shown in Fig. 5. It can be seen that when there is no defect in the pipe, the real and imaginary parts of the axial magnetic field are both near zero. When the annular 


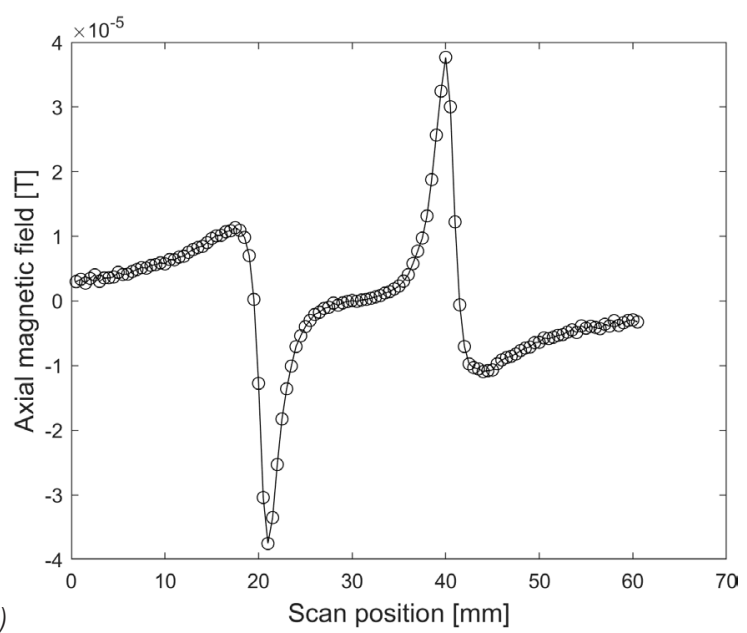

a)

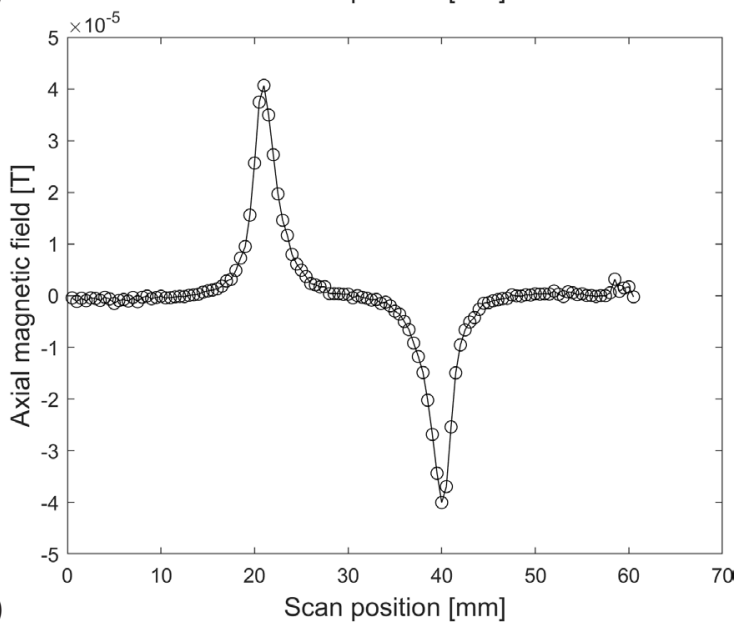

Fig. 5. Real and imaginary parts of the characteristic signal; a) real part, and b) imaginary part

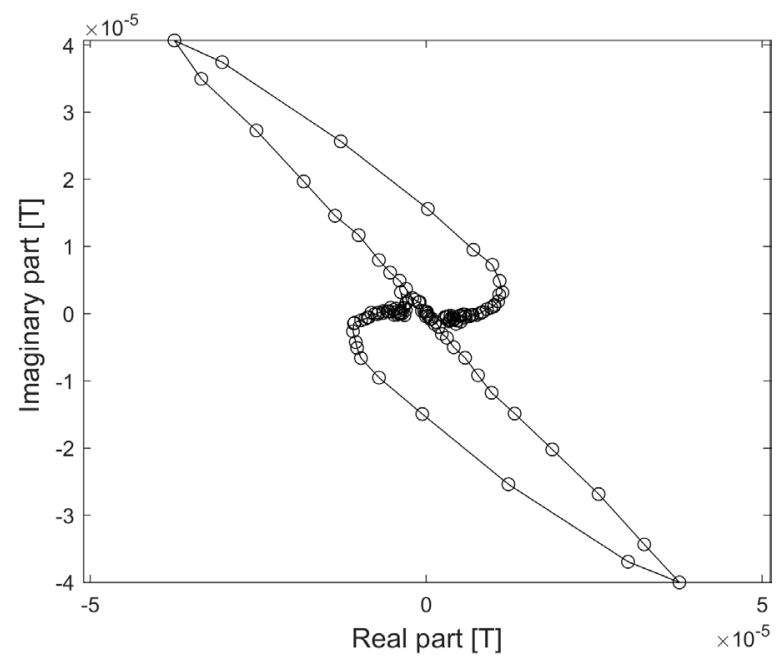

Fig. 6. Real and imaginary part of the characteristic signal plotted as a Lissajous pattern in the impedance plane

eddy current flows to the left, a wave trough is generated. When it flows to the right, a wave crest is generated. The real and imaginary parts of the axial magnetic field are plotted as a Lissajous pattern in the impedance plane, as shown in Fig. 6. Note that, as demonstrated in the experimental section, such a magnetic field can be measured by a circumferential bobbin coil around the pipe and used for defect evaluation.

\section{FE MODEL-BASED ANALYSIS ON THE INFLUENCING FACTORS}

Influencing factors of the RoFEC technique were then studied using the FE model, and the findings can be used to guide subsequent experiments.

\subsection{Effects of Pipe Tilt}

Considering that the pipe tilt during the actual inspection process, which will affect the inspection results, it is necessary to study the effects of the pipe tilt on defect detection. Fig. 7 shows the case in which the defective side of the pipe is inclined to the $x$-axis.

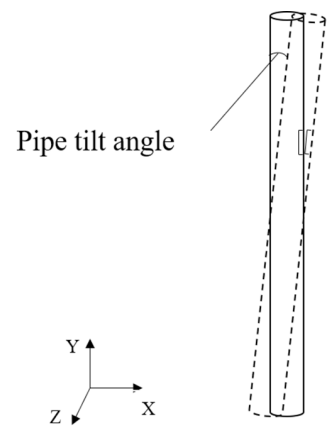

Fig. 7. Schematic diagram of pipe inclining along the $x$-axis

When the inclination angle of the pipe is set to $3^{\circ}$, $6^{\circ}, 9^{\circ}, 12^{\circ}$ and $15^{\circ}$, the defects are detected and the characteristic signals (real part of the axial magnetic field $1 \mathrm{~mm}$ above the defect) are extracted, as shown in Fig. 8. It can be seen from Fig. 8a that when the pipe is inclined to the $\mathrm{x}$-axis, the value of the axial magnetic field peak is greater than the value of the trough because the defect is coming closer to the excitation windings. When the tilt angle is greater than $12^{\circ}$, the change is more significant. This is because the closer it is to the excitation windings, the more drastic the magnetic field changes. When the pipe is inclined to the z-axis, since the pipe is not inclined to the side of the inspection point, the peak value of the axial magnetic field is basically unchanged, as shown in Fig. 8b. 
It can also be seen from both Figs. 8a and $\mathrm{b}$ that, no matter to which direction the pipe is tilted, the values of the axial magnetic field at defect-free positions (the starting point of each curve) are no longer zero. This is due to the change of the spatial background magnetic field caused by the inclination of the pipe, which generates the axial component of the magnetic field.

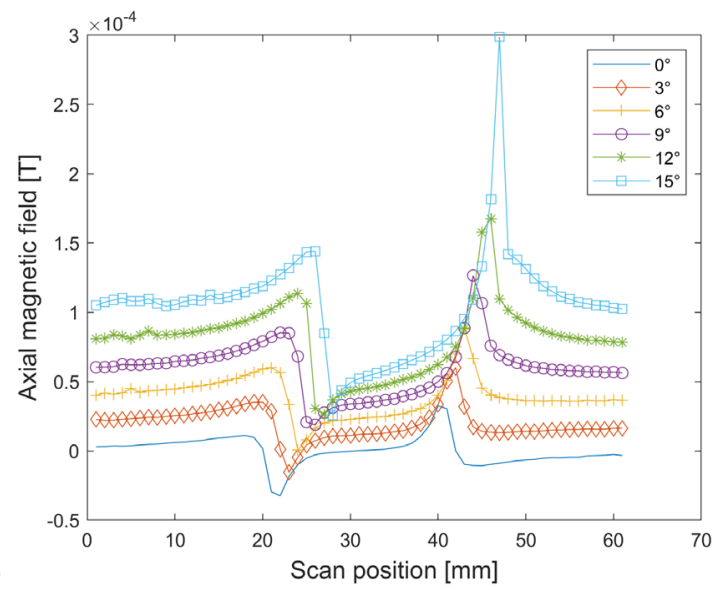

a)

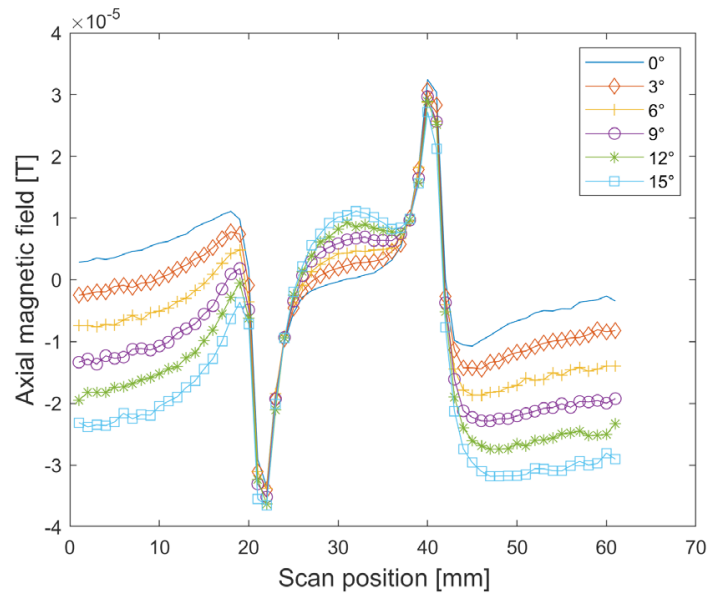

Fig. 8. Characteristic signals (real part of the axial magnetic field) with different defect tilt angles; a) tilt towards the $x$-axis, and b) tilt towards the $z$-axis

\subsection{Effects of Circumferential Location of the Defect}

Because possible defects may appear at any position of the pipe, it is necessary to study how the resultant magnetic field will vary with different circumferential locations of the defect. In the FE model, 8 axial defects with exactly the same size but at different circumferential locations (at angles of 0 degrees, 45 degrees, 90 degrees, 135 degrees, 180 degrees, 225 degrees, 270 degrees and 315 degrees with respect to the reference point) were scanned. The eight characteristic signals, due to the defects, are extracted and plotted as Lissajous patterns in the impedance diagram, as shown in Fig. 9. Note that, to avoid signal overlapping, only the first half of the characteristic signals are plotted for each Lissajous pattern. It can be seen from Fig. 9 that with the different circumferential locations of the defect on the outer wall of the pipe the angle of the Lissajous patterns also change accordingly, based on which the circumferential location of the defect can be determined. It can also be noted that all the Lissajous patterns are of similar size.

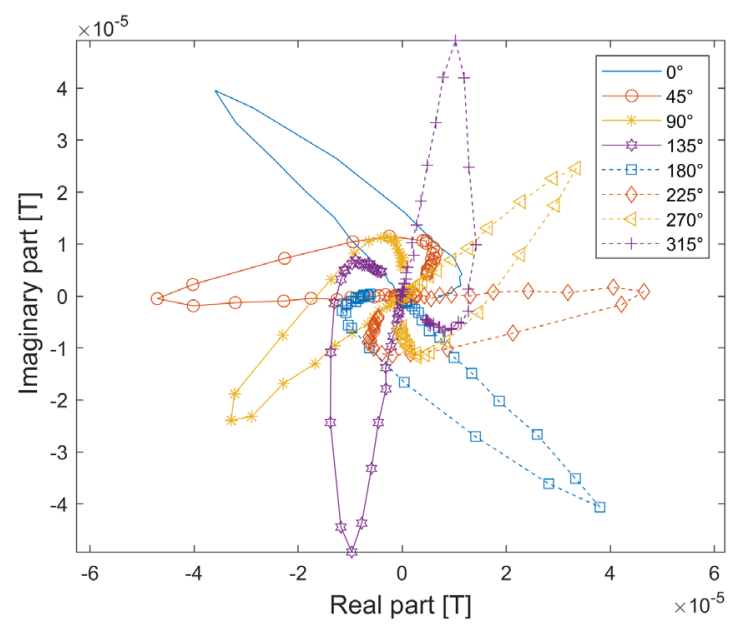

Fig. 9. Lissajous Patterns for defects at different circumferential locations

\subsection{Effects of Defect Orientation}

The effects of the orientations of the defect on the characteristic signal of the rotating electromagnetic field were also studied. Seven defects with 0-degree, 15-degree, 30-degree, 45-degree, 60-degree, 75-degree, and 90-degree orientations were introduced on the outer wall of the pipe in the FE model. As shown in Fig. 10, the defects are centred at the same point.

\section{Defects of}

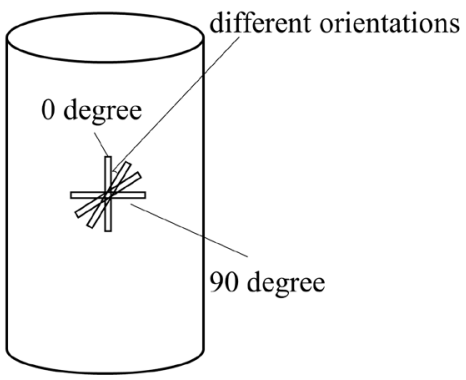

Fig. 10. Schematic diagram of defects of different directions 
Fig. 11 shows the axial magnetic field of different defect orientations. It can be seen that only when the direction of the defect is 0 degrees or 90 degrees, the peak and trough of the axial magnetic field curve have the same absolute value. As the defect orientation changes from 0 degrees to 90 degrees, the absolute value of the peak of the axial magnetic field curve is greater than that of the trough. This phenomenon is most significant when the defect orientation is 45 degrees. The reason is that when the defect orientation is 45 degrees, the defect is affected by both the axial component and the circumferential component of the eddy current. At the same time, the distance between the peak and trough of the axial magnetic field keeps becoming closer. This set of FE models demonstrated that the encircling RoFEC technique is sensitive to defects of all orientations.
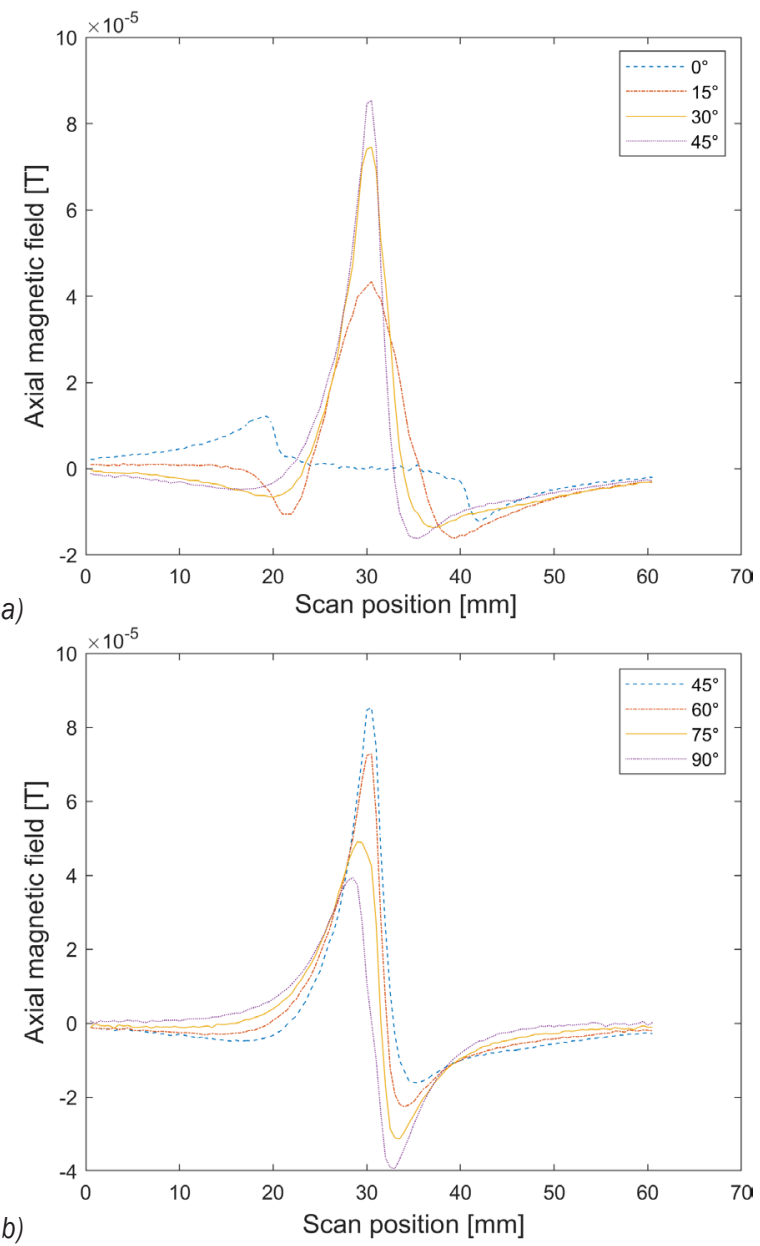

Fig. 11. Axial magnetic field of defects in different directions; a) $0^{\circ}$ to $45^{\circ}$, and b) $45^{\circ}$ to $90^{\circ}$

\subsection{Effects of Defect Size}

The effects of the defect size on the characteristic signal, including the defect's length, width, and depth, are also studied to lay the foundation for the quantification method of defects in the future.

It can be seen from Fig. 12a that with the length of the defect varies from $10 \mathrm{~mm}$ to $50 \mathrm{~mm}$, and the distance between the peak and trough of the axial magnetic field curve also changes. The Lissajous patterns are then drawn, as shown in Fig. 12b. The sizes of the Lissajous patterns are also related to the length of each defect.

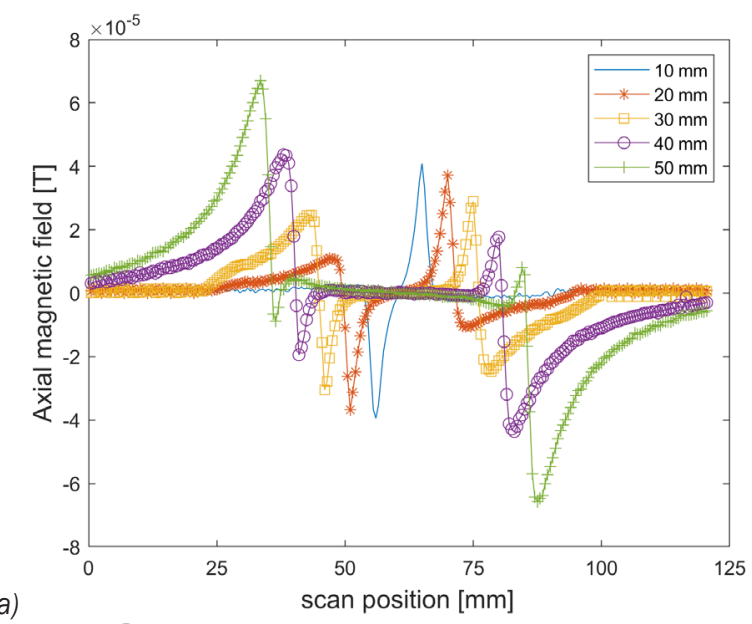

a)

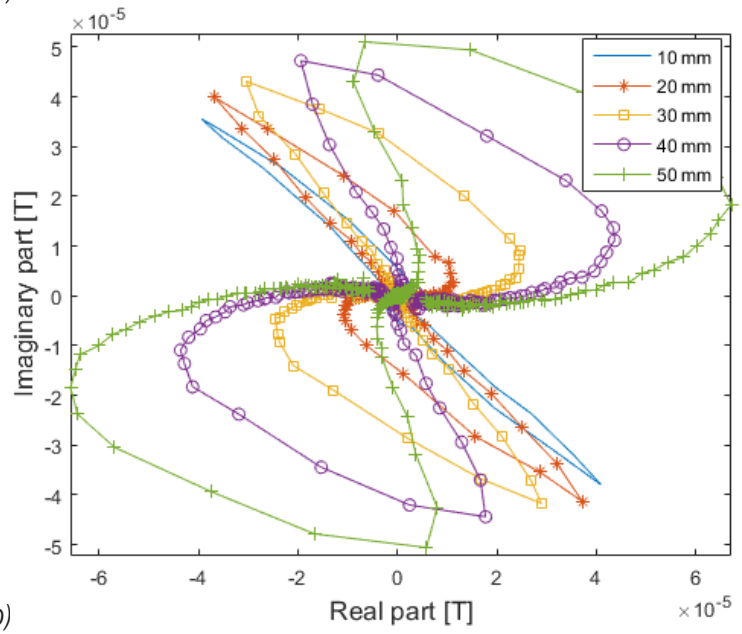

Fig. 12. a) Axial magnetic field of defects with different lengths, and b) the Lissajous patterns in the impedance plane

The effects of defect widths were also studied. The width of the defect varies from $1 \mathrm{~mm}$ to $4 \mathrm{~mm}$, and the axial magnetic field is also extracted, as shown in Fig. 13a. It can be seen from the figure that the peak and trough of the axial magnetic field curve increase with the increase of the width of the defect. 

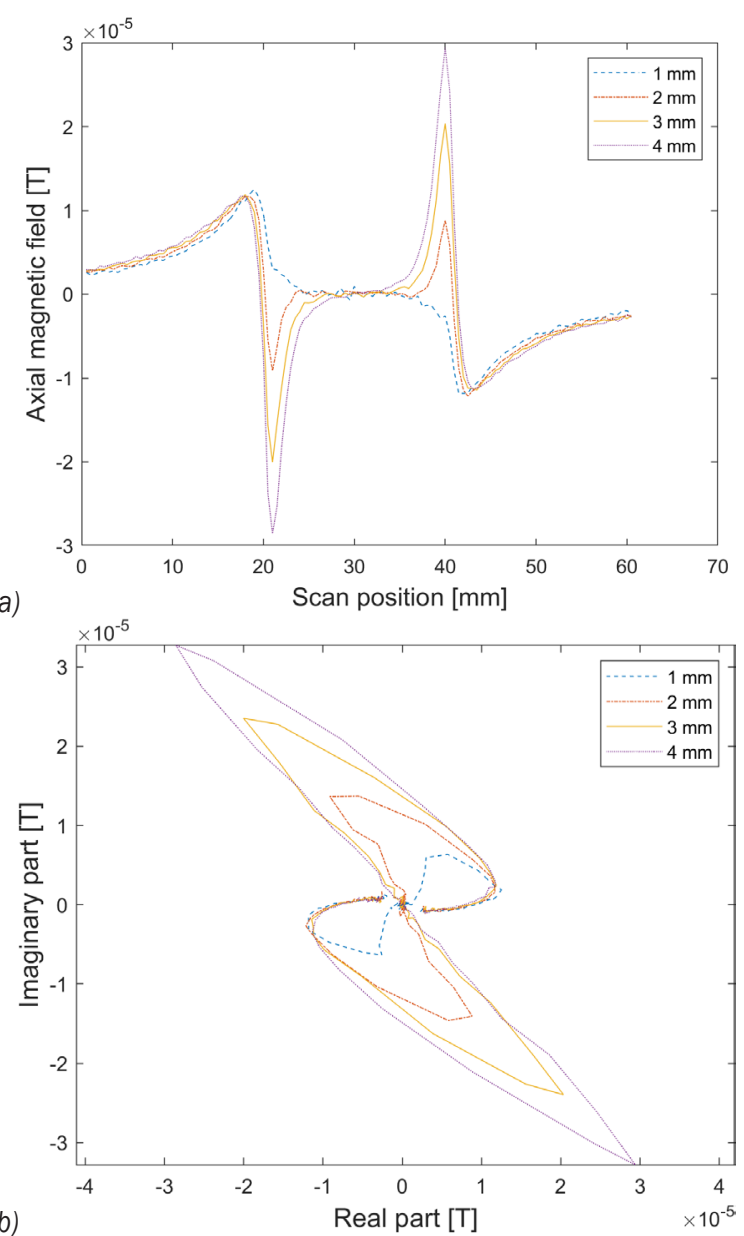

Fig. 13. a) Axial magnetic field of defects with different widths and b) The Lissajous patterns in the impedance plane

Fig. 13b shows the Lissajous patterns of defects with different widths. The sizes of the Lissajous patterns also increase with defect width.

Finally, the effects of defect depth on the characteristic signal is studied. Fig. 14a shows the axial magnetic field of defects with different depths. The depth of the defects varies from $1 \mathrm{~mm}$ to $4 \mathrm{~mm}$. The peak value of the axial magnetic field curve is basically unchanged, leading to a less significant change in the Lissajous patterns in Fig. 14b compared to the previous two cases.

\section{EXPERIMENTS}

An encircling RoFEC system is built to verify the feasibility of the proposed method. The hardware part of the system mainly includes a signal generator, voltage amplifier, lock-in amplifier, the encircling RoFEC probe, data acquisition card and personal
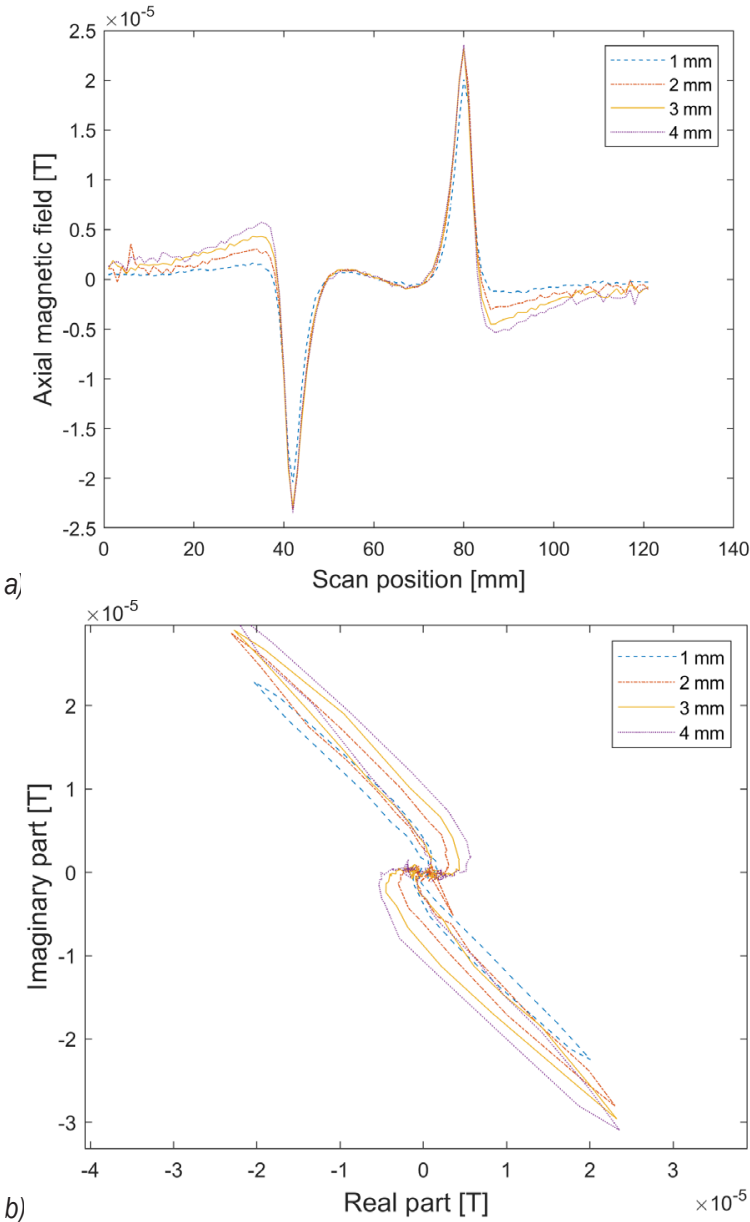

Fig. 14. a) Axial magnetic field of defects with different depths, and b) the Lissajous patterns in the impedance plane

computer. It can run independently and complete specific functions. The software part was developed in LabVIEW, including a human-computer interface, data acquisition module, and data processing module. The overall block diagram of the detection system is shown in Fig. 15.

The encircling RoFEC probe is comprised of six excitation windings (shown in Fig. 16a) with the same specifications as the ones used in the FE model and a 50-turn bobbin coil ( $52 \mathrm{~mm}$ in diameter) placed in the centre of the excitation windings around the pipe (shown in Fig. 16b).

Such a bobbin pickup coil in the plane of the rotating field is only sensitive to variations in the axial component of the magnetic field. The coil is self-nulling since when integrated over one circle, the voltage induced in the bobbin coil will be zero at a defect-free position. In addition, as demonstrated in experiments, the phase of the induced voltage in 


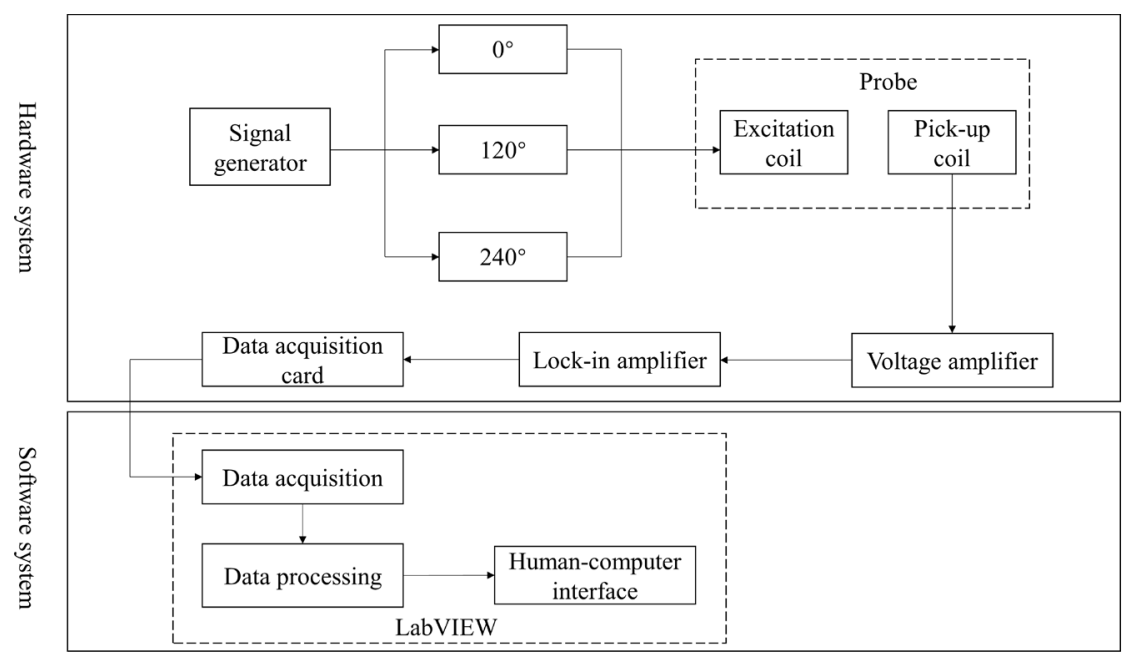

Fig. 15. Block diagram of the encircling RoFEC system

the pickup coil is correlated with the circumferential location of the defect in the pipe. Also, under this measurement scheme, the diameter of the bobbin pickup coil is preferably large, so the bobbin pickcoil for the encircling RoFEC probe can have a much bigger diameter than the pipe, leading to a bigger tolerable lift-off distance.

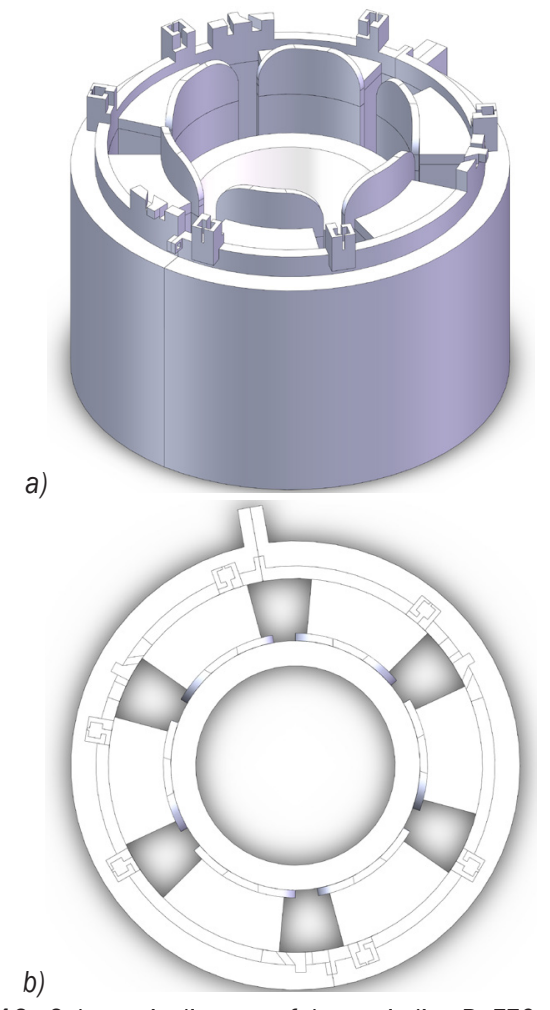

Fig. 16. Schematic diagram of the encircling RoFEC probe; a) the excitation windings and b) top view of the probe with the bobbin pickup coil in the centre
The signal generator provided the three-phase sinusoidal excitation $(10 \mathrm{kHz}, 20 \mathrm{~V}$ pk-pk) to generate the rotating electromagnetic field. The voltage amplifier, lock-in amplifier, and data acquisition card were used to process and record the characteristic signal due to defect. The characteristic signal was collected in the computer for further processing and display. In order to realize the axial scanning of the tested pipe, a pipe-scanning platform was also designed. The platform is composed of three sliders, two height adjustment devices, and a slide rail. Two sliders are located at the two ends of the slide rail and are used to fix the two ends of the pipe. The middle slider is used to control the movement of the probe to implement the circumferential scan. The height adjustment device is fixed on the slide rails at both ends for adjusting the height of the pipe. Fig. 17 is the photo of the whole RoFEC system.

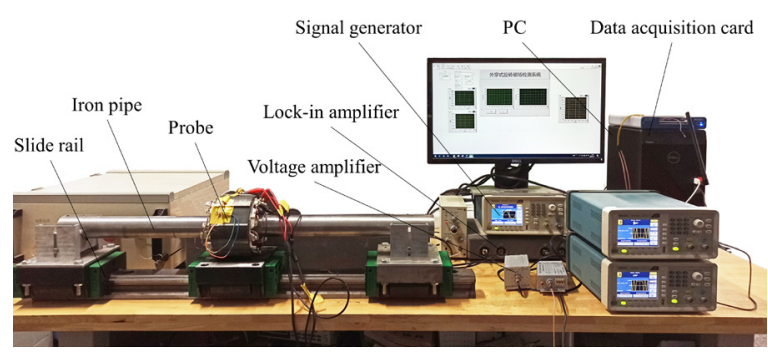

Fig. 17. RoFEC system

A steel pipe with a $50 \mathrm{~mm}$ outer diameter and $40 \mathrm{~mm}$ inner diameter was used as a specimen. Two artificial slots $(25 \mathrm{~mm} \times 1 \mathrm{~mm} \times 3 \mathrm{~mm})$ were machined on the outer wall of the pipe, as shown in Fig. 18. 
Axial crack Circumferential crack

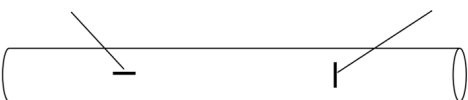

Fig. 18. Schematic diagram of specimen

Firstly, the axial defect in the pipe was scanned by the encircling RoFEC system, and the results are shown in Fig. 19. Fig. 19a is the real part of the voltage across the pickup coil, and Fig. 19b is the imaginary part. At the defect-free position, i.e., the starting point of the voltage curve, the measured voltage is basically stable at a near-zero value. When the annular eddy current encounters a crack-like defect, it bypasses each end of the defect in opposite directions, so the measured voltage will have one crest and one trough; this is consistent with the results obtained in the FE model.

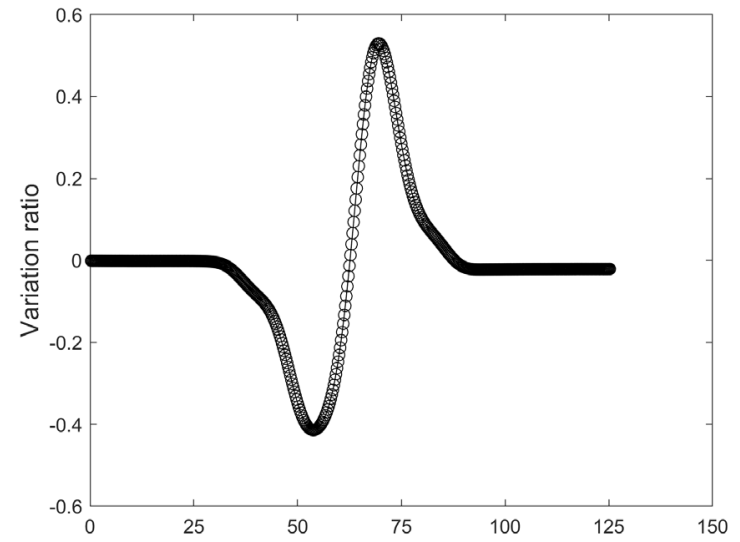

a)

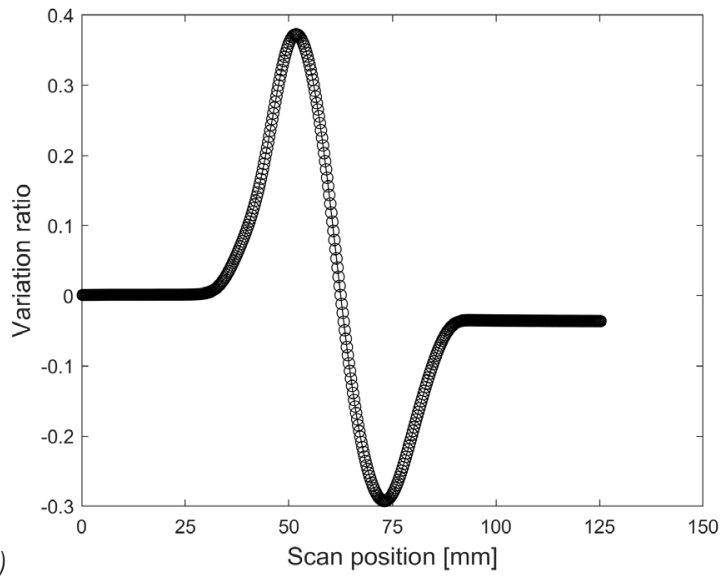

Fig. 19. Measured voltage for the axial defect; a) real part, b) imaginary part

The real and imaginary parts of the voltage are then plotted in an impedance plane, as shown in Fig. 20 . The plot in the impedance plane integrates the real and imaginary parts and is seen as a Lissajous pattern.

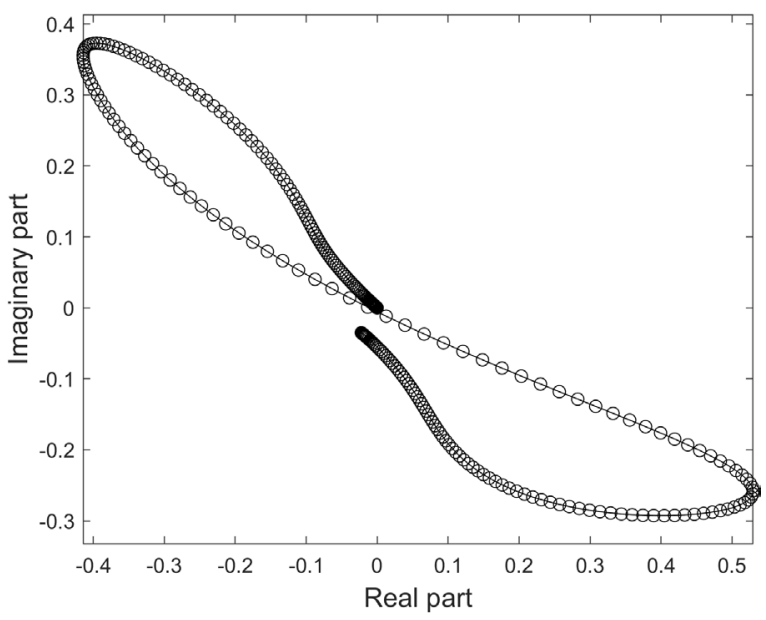

Fig. 20. Impedance plane plot for the axial defect

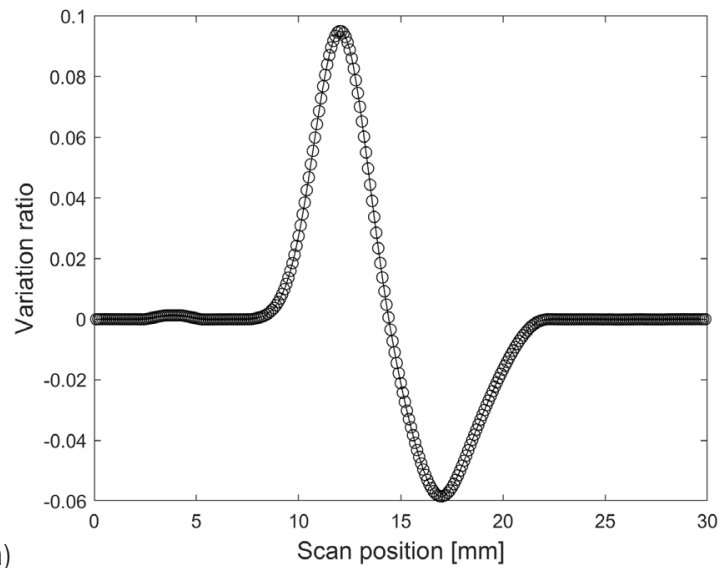

a)

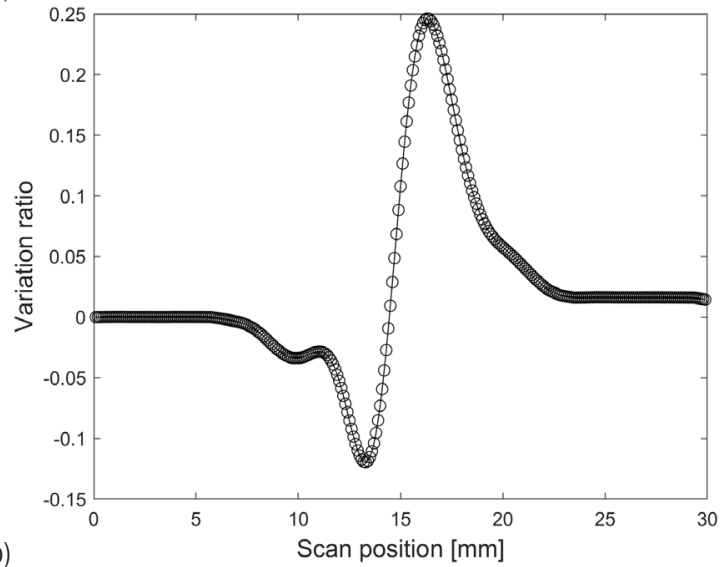

Fig. 21. Measured voltage for the circumferential defect; a) real part, and b) imaginary part

The RoFEC system was then used to detect the circumferential defect, and the results are shown in Fig. 21. Fig. 21a is the real part of the voltage across the pickup coil, and Fig. $21 \mathrm{~b}$ is the imaginary part. 
Comparing Figs. 19 and 21, it can be seen that, as indicated by the FE simulation results (Fig. 11), the variation range of the measured voltage is bigger for a circumferential defect.

The real and imaginary parts of the voltage are then plotted in an impedance plane, as shown in Fig. 22. The plot in the impedance is also seen as a Lissajous pattern. The above test results are consistent with the results of the FE model and proved that the RoFEC technique can detect defects in any direction.

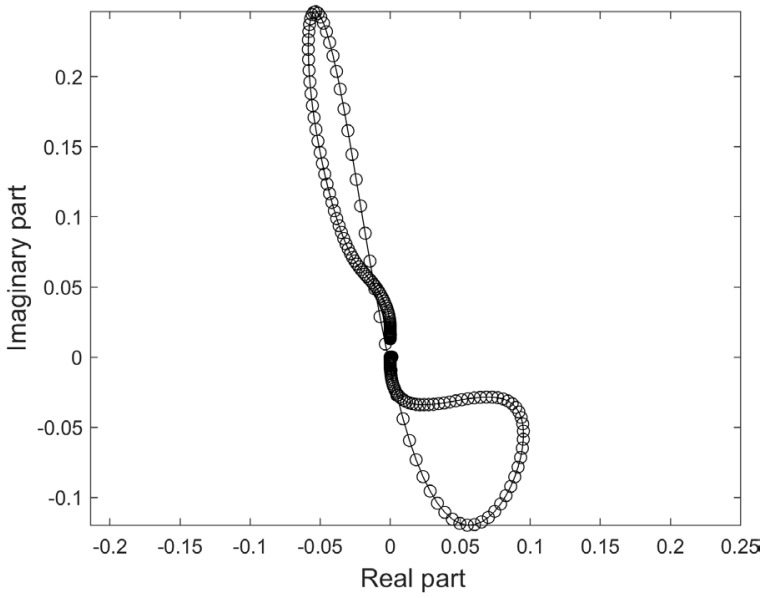

Fig. 22. Impedance plane plot for the circumferential defect

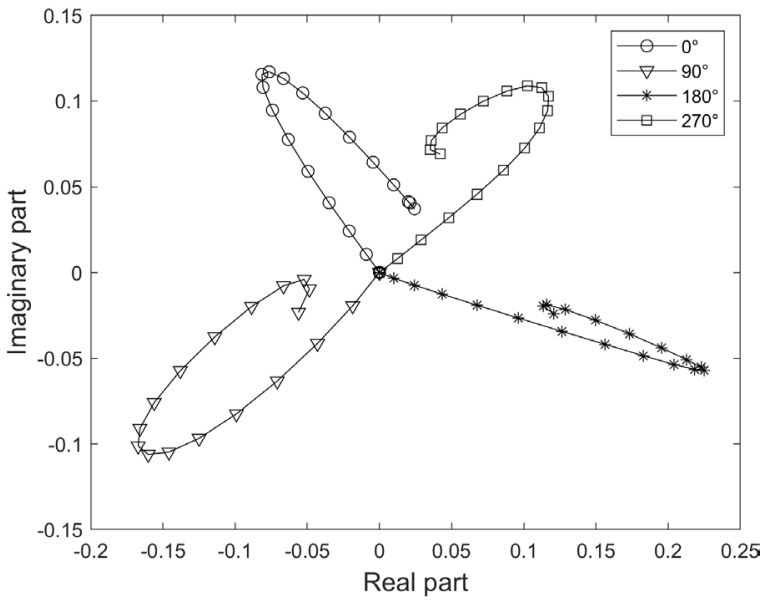

Fig. 23. Impedance plane plots with the defect at different circumferential locations

The ability to determine the defect's circumferential location was also verified using the axial defect. In the designed experiments, the pipe was rotated by different angles, and line scans with the axial crack being at 0 degrees, 90 degrees, 180 , and 270 degrees with respect to the reference point were performed. The resultant impedance plane plots are shown in Fig. 23. It can be seen that when the circumferential location of the defect on the outer wall of the pipe is different, the angle of the Lissajous pattern changes accordingly. This is also consistent with the FE result, which verified that the encircling RoFEC technique can accurately determine the circumferential location of the defect.

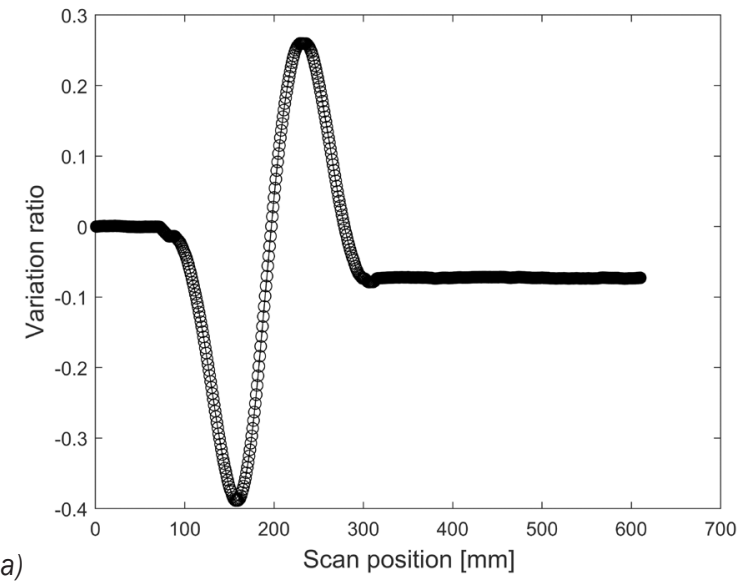

a)
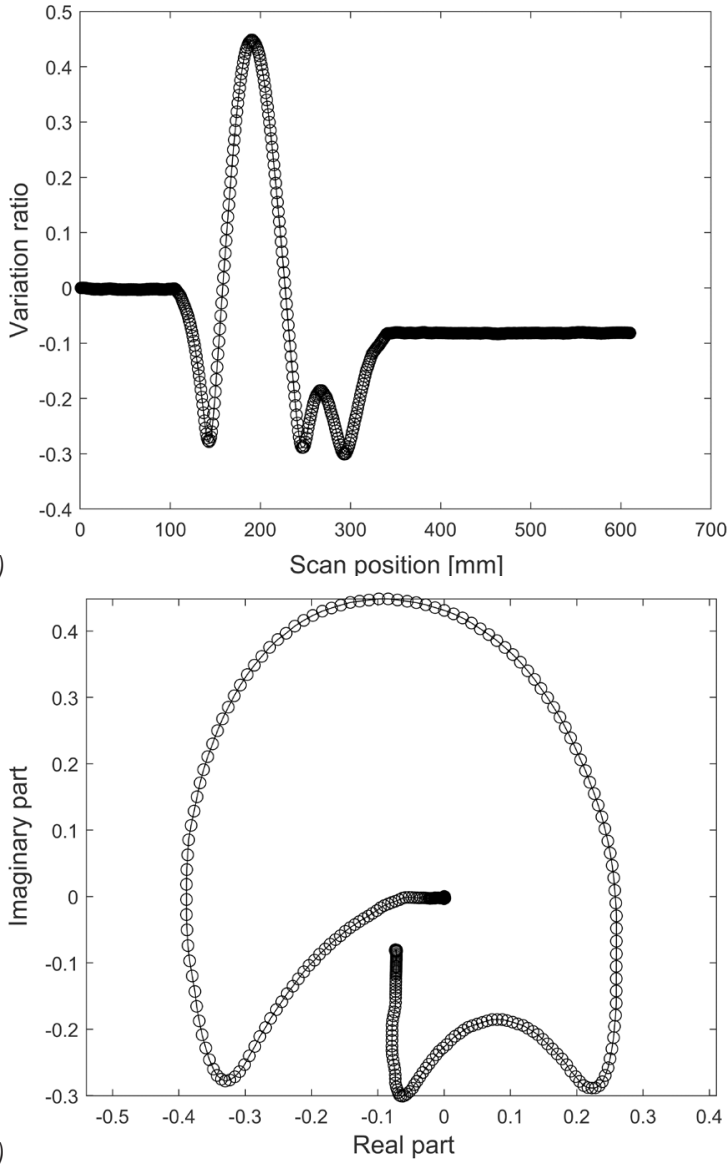

Fig. 24. Experimental results for the circumferential defect

a) Real part of measured voltage b) Imaginary part of measured voltage, and c) Impedance plane plot 
Another experiment on a specimen with a smaller crack was also conducted to test the inspection ability for smaller defects. The specimen is a steel pipe with a $50 \mathrm{~mm}$ outer diameter and $40 \mathrm{~mm}$ inner diameter. One narrower axial slot $(20 \mathrm{~mm} \times 0.3 \mathrm{~mm} \times 1.5 \mathrm{~mm})$ compared to the ones shown in Fig. 18 was machined on the outer wall of the pipe. The encircling RoFEC system scanned the axial slot in the pipe, and the results are shown in Fig. 24. Fig. 24a is the real part of the voltage across the pickup coil, Fig. $24 \mathrm{~b}$ is the imaginary part, and Fig. 24c is the impedance plane plot. Although the small crack was also detected, the indications are less informative than the bigger defect case. In particular, the impedance plot (shown in Fig. 24c) does not form a "proper" Lissajous pattern and cannot be used to locate the circumferential location of the defect. Such an experiment indicates that the current instrument for the encircling RoFEC technique is only at a proof-of-concept stage, and significant improvement is required.

\section{CONCLUSIONS}

This paper proposed an encircling RoFEC technique to detect outer wall defects on steel pipes. The working principle of the RoFEC technique was introduced, and the effects of pipe tilt, defect circumferential location, defect orientation and defect size on the inspection performance were analysed in detail using the $\mathrm{FE}$ method. An encircling RoFEC system was also built to verify the FE results. The test results showed that the RoFEC technique can effectively detect both axial and circumferential defects. In addition, when the circumferential locations of the defect are different, the angle of the Lissajous pattern in the impedance plane can be used to determine the circumferential location accurately.

Compared with the existing approaches for RoFEC testing from inside the tube [21] and [22], in which cases the targeted tubes are nonferrous materials (e.g. Inconel 600), the encircling RoFEC technique described in this paper focused on the inspection of pipes made of carbon steel, a magnetically permeable material that is more difficult for eddy current related techniques. Another major difference between the encircling and feedthrough approaches is the single bobbin pickup coil. Although the single bobbin pickup coil is used in both approaches, the preferences of the coil diameter may be different. For the feed-through approach, the bobbin coil is preferably close to the inner wall to eliminate the lift-off effect, while for the encircling approach, the coil diameter is a design concern. A bobbin coil close to the outer wall of the pipe may not be an optimal choice, and a careful design is required.

It should be noted that this paper aims to demonstrate the feasibility of the encircling RoFEC technique; at present, the encircling RoFEC technique remains at a very early stage, and the current instrument needs to be improved significantly to be able to detect and quantify tiny flaws. Probe design and other influential factors, e.g. lift-off distance of the excitation windings, the diameter of bobbin pick up coil, motion-induced eddy current, and permeability variation due to $\mathrm{AC}$ magnetic field, are also worth investigating in further studies.

\section{ACKNOWLEDGEMENTS}

This work was funded by the National Natural Science Foundation of China (No. 52075549), the Major Scientific and Technological Projects of CNPC (ZD2019-183-004), and the Fundamental Research Funds for the Central Universities (19CX02023A).

\section{REFERENCES}

[1] Zhou, Z., Tan, J., Wan, F., Peng, B. (2019). Improvement and determination of the influencing factors of coiled tubing fatigue life prediction. Advances in Mechanical Engineering, vol. 11, no. 9, art. ID 2072157101, Dol:10.1177/1687814019880131.

[2] Brockhaus, S., Ginten, M., Klein, S., Teckert, M., Stawicki, O., Oevermann, D., Meyer, S., Storey, D. (2014). In-line inspection (ILI) methods for detecting corrosion in underground pipelines, in Underground Pipeline Corrosion, Orazem, M.E. (ed.), Woodhead Publishing, p. 255-285, D0l:10.1533/978085709 9266.2.255.

[3] Rifai, D., Abdalla, A., Razali, R., Ali, K., Faraj, M. (2017). An eddy current testing platform system for pipe defect inspection based on an optimized eddy current technique probe design. Sensors, vol. 17, no. 3, art. ID 579, D0l:10.3390/s17030579.

[4] Yuan, X.A., Li, W., Chen, G., Yin, X., Ge, J. (2017). Circumferential current field testing system with TMR sensor array for non-contact detection and estimation of cracks on power plant piping. Sensors and Actuators A: Physical, vol. 263, p. 542-553, D0I:10.1016/j.sna.2017.07.029.

[5] Takagi, T., Huang, H., Fukutomi, H., Tani, J. (1998). Numerical evaluation of correlation between crack size and eddy current testing signal by a very fast simulator. IEEE Transactions on Magnetics, vol. 34, no. 5, p. 2581-2584, DOI:10.1109/20.717596.

[6] Kim, H.M., Heo, C.G., Cho, S.H., Park, G.S. (2018). Determination scheme for accurate defect depth in underground pipeline inspection by using magnetic flux leakage sensors. IEEE Transactions on Magnetics, vol. 54, no. 11, p. 1-5, D0I:10.1109/TMAG.2018.2853618.

[7] Pham, H.Q., Tran, B.V., Doan, D.T., Le, V.S., Pham, Q.N., Kim, K., Kim, C., Terki, F., Tran, Q.H. (2018). Highly sensitive planar hall magnetoresistive sensor for magnetic flux leakage 
pipeline inspection. IEEE Transactions on Magnetics, vol. 54, no. 6, p. 1-5, DOI:10.1109/TMAG.2018.2816075.

[8] Liu, T., Pei, C., Cai, R., Li, Y., Chen, Z. (2020). A flexible and noncontact guided-wave transducer based on coils-only EMAT for pipe inspection. Sensors and Actuators: A. Physical, vol. 314, art. ID 112213, D0I:10.1016/j.sna.2020.112213.

[9] Liu, Z., Li, A., Zhang, Y., Deng, L., Wu, B., He, C. (2020). Development of a directional magnetic-concentrator-type electromagnetic acoustic transducer for ultrasonic guided wave inspection. Sensors and Actuators A: Physical, vol. 303, p. 111859, DOI:10.1016/j.sna.2020.111859.

[10] Yang, Q., Xie, S., He, K., Chen, Y., Chen, Z., Uchimoto, T., Takagi, T. (2021). A novel circumferential eccentric eddy current probe and its application for defect detection of small-diameter tubes. Sensors and Actuators A: Physical, vol. 331, art. ID 113023, D0l:10.1016/j.sna.2021.113023.

[11] Zhao, Y., Qi, P., Xie, Z., Bai, P., Chen, H., Xie, S., Liao, S., Chen, Z. (2020). A new array eddy current testing probe for inspection of small-diameter tubes in Tokamak fusion devices. Fusion Engineering and Design, vol. 157, art. ID 111627, DOI:10.1016/j.fusengdes.2020.111627.

[12] Alpeisov, E.A., Adzhanov, A.U., Sarsembieva, E.K. (2017). Application of a three-phase sinusoidal current to produce a rotating magnetic field. International Siberian Conference on Control and Communications, p. 1-3, D0l:10.1109/ SIBCON.2017.7998480.

[13] Ye, C., Huang, Y., Udpa, L., Udpa, S.S. (2016). Differential sensor measurement with rotating current excitation for evaluating multilayer structures. IEEE Sensors Journal, vol. 16, no. 3, p. 782-789, DOI:10.1109/JSEN.2015.2488289.

[14] Ye, C., Udpa, L., Udpa, S. (2016). Optimization and validation of rotating current excitation with GMR array sensors for riveted structures inspection. Sensors, vol. 16, no. 9, art. ID 1512, D0l:10.3390/s16091512.

[15] Yang, G., Dib, G., Udpa, L., Tamburrino, A., Udpa, S.S. (2015). Rotating field EC-GMR sensor for crack detection at fastener site in layered structures. IEEE Sensors Journal, vol. 15, no. 1, p. 463-470, DOI:10.1109/JSEN.2014.2341653.

[16] Xin, J., Lei, N., Udpa, L., Udpa, S.S. (2011). Non-destructive inspection using rotating magnetic field eddy-current probe. IEEE Transactions on Magnetics, vol. 47, no. 5, p. 1070-1073, DOI:10.1109/TMAG.2011.2108996.
[17] Ye, C., Huang, Y., Udpa, L., Udpa, S.S. (2016). Novel rotating current probe with GMR array sensors for steam generate tube inspection. IEEE Sensors Journal, vol. 16, no. 12, p. 4995 5002, DOI:10.1109/JSEN.2016.2556221.

[18] Ye, C., Xin, J., Su, Z., Udpa, L., Udpa, S.S. (2015). Novel transceiver rotating field non-destructive inspection probe. IEEE Transactions on Magnetics, vol. 51, no. 7, p. 1-6, DOI:10.1109/TMAG.2015.2390144.

[19] Li, Y., Gao, X., Zhang, Y., You, D., Zhang, N., Wang, C., Wang, C. (2020). Detection model of invisible weld defects by magnetooptical imaging at rotating magnetic field directions. Optics \& Laser Technology, vol. 121, art. ID 105772, D0l:10.1016/j. optlastec.2019.105772.

[20] Gao, X., Du, L., Ma, N., Zhou, X., Wang, C., Gao, P.P. (2019). Magneto-optical imaging characteristics of weld defects under alternating and rotating magnetic field excitation. Optics \& Laser Technology, vol. 112, p. 188-197, D0l:10.1016/j. optlastec.2018.11.005.

[21] Xin, J., Lei, N., Udpa, L., Udpa, S.S. (2013). Rotating field eddy current probe with bobbin pickup coil for steam generator tubes inspection. NDT \& E International, vol. 54, p. 45-55, DOI:10.1016/J.ndteint.2012.12.002.

[22] Lei, N., Udpa, L., Udpa, S., Zeng, Z. (2010). Rotating field eddy current (RoFEC)-probe for steam generator inspection. International Journal of Applied Electromagnetics and Mechanics, vol. 33, p. 1279-1285, D0I:10.3233/JAE-2010. 1250.

[23] Grimberg, R., Udpa, L., Savin, A., Steigmann, R., Vizureanu, P., Bruma, A., Udpa, S.S. (2008). Remote field eddy current control using rotating magnetic field transducer: application to pressure tubes examination. Research in Non-destructive Evaluation, vol. 19, no. 4, p. 202-218, DOl:10.1080/09349840802190090.

[24] Qka, M., Enokizono, M. (2020). A detection of backside crack using rotational magnetic flux sensor with search coils. IEEE Transactions on Magnetics, vol. 32, no. 5, p. 4968-4970, Dol:10.1109/20.539304.

[25] Koyama, K., Hoshikawa, H., Sakon, T. (2011). Study on metal detection with electromagnetic induction probe utilizing rotating magnetic field. Electrical Engineering in Japan, vol. 177, no. 4, p. 1-11, D0I:10.1002/eej.21178. 